\title{
Does Sandblasting Improve Bond Strength between Nano-ceramic Resin and Two Different Luting Composites?
}

Augusti Davide, Augusti Gabriele*, Cerutti Francesca and Re Dino

Department of Biomedical, Surgical and Dental Sciences, University of Milan, Division of Oral Rehabilitation, Istituto Stomatologico Italiano, Milan, Italy

\begin{abstract}
Purpose: To assess the influence of a sandblasting surface treatment on the shear bond strength (SBS) between a recently introduced Resin Nano-Ceramic (RNC) CAD/CAM material and two resin cements.

Methods: 40 RNC blocks were divided into two groups ( $n=20)$ : a control (C) and a test group (S_50) to which air abrasion $(50 \mu \mathrm{m}, 0.28 \mathrm{MPa}$, and $10 \mathrm{~s})$ was applied. Each group was divided into two subgroups $(\mathrm{n}=10)$ according to the applied luting material: a self-etch dual-cure resin cement (LP) and a dual-cure resin cement (LR). SBSs were determined with a universal testing machine at a crosshead speed of $0.5 \mathrm{~mm} / \mathrm{min}$. Fracture patterns were evaluated by a stereomicroscope (25x) and classified into adhesive, cohesive and mixed. One-way ANOVA test ( $\alpha=.01)$ was used to establish differences between groups, considering SBS as dependent and the type of surface treatment as independent variable.
\end{abstract}

Results: Mean SBS values (MPa) and standard deviations (SD) for the experimental groups were: [C-LP]: 8.68 (1.16); [C-LR]: 15.32 (3.93); [S 50-LP]: 13.91 (2.58); [S 50-LR]: 15.77 (4.12). A statistically significant difference $(p<0.001)$ was found between $[\bar{C}-L P]$ and the other three groups. Sandblasting positively affected $(p<0.01)$ the selfadhesive cement adhesion to nano-ceramic resin.

Group C LP showed $100 \%$ of adhesive fractures, while S 50 LP had some cohesive (20\%) and mixed fractures (10\%). The fracture pattern of group C_LR was $60 \%$ cohesive, $20 \%$ adhesive and $20 \%$ mixed, while group S_50-LR showed $100 \%$ of cohesive failure.

Conclusion: Within the limitations of this study, sandblasting of the RNC surface is recommended when the tested self-adhesive resin cement is used for luting, in order to significantly improve bond strength values. For the luting agent LR - associated with a universal adhesive system - satisfactory adhesion values were achieved regardless an air abrasion pre-treatment.

Keywords: Resin nanoceramic; CAD/CAM; Luting cements; Adhesion

\section{Introduction}

Contemporary dental restorations developed by the use of Computer-Aided Design/Computer-Aided Manufacturing (CAD/ CAM) technology are frequently adopted for providing high-quality, long-term oral rehabilitations in prosthodontics [1]. The design and manufacturing of the restoration can be made either in the dental laboratory or directly in the dental office, with the advantage of reduced treatment time $[1,2]$. Several CAD/CAM systems are available to process reinforced glass-ceramics, high-strength (i.e: densely sintered zirconium oxide or alumina) all-ceramics and resin-based or composite materials $[3,4]$. Based on the industrial standardized polymerization of $\mathrm{CAD} / \mathrm{CAM}$ resin blanks under high pressure and temperature, significantly higher physical and mechanical properties can be achieved compared to conventionally polymerized materials $[5,6]$. Not only improved mechanical behavior but also fewer discolorations and higher abrasion resistance in relation to the conventional polymerized resins were obtained $[7,8]$. Recently, a new group of CAD/CAM machinable blocks made of composite resin and nanofillers has been introduced to the market [9-11]. In particular, LAVA Ultimate ${ }^{\mathrm{TM}}(3 \mathrm{M}$ Espe) blanks are composed of $80 \mathrm{wt} \%$ zirconia/silica nanoceramic particles embedded in a highly cross-linked resin matrix (20 wt\%); it contains silica nanomers $(20 \mathrm{~nm})$, zirconia nanomers $(4$ to $11 \mathrm{~nm})$, nanocluster particles derived from the nanomers ( 0.6 to $10 \mu \mathrm{m})$, silane coupling agent, and resin matrix. Due to its peculiar composition, this kind of dental material has been named Resin Nano Ceramic (RNC) and offers the benefits of both glass ceramic and resin materials [11].
Additional features are an adequate flexural strength (about 230MPa) and satisfactory resistance to fracture loads [12], and a reduced wear produced to opposing enamel when compared to traditional glass ceramics [8]. According to the manufacturer's guidelines, the main clinical applications of the RNC CAD/CAM material include minimally-invasive partial indirect restorations like inlays, onlays and overlays to replace lost tooth structure; in addition, long-term single anterior and posterior crowns can also be delivered [13]. On the other hand, traditional high-strength densely sintered ceramics (i.e: made of yttria-stabilized tetragonal zirconia polycrystal - Y-TZP), with flexural strength in the range of $800-1000 \mathrm{MPa}$, are indicated for medium or long-span posterior prosthetic restorations, like three or four-unit fixed partial dentures $[14,15]$. Adhesion between the tooth structure and the restoration is one of the most important factors determining the success of a restoration: despite the properties of the material, adhesive interface is the locus minoris resistentiae of a luted

*Corresponding author: Augusti Gabriele, Division of Oral Rehabilitation, Schoo of Dentistry, Prosthodontics Division, University of Milan, Via Pace 21, 20122 Milan, Italy, Tel: 3902503111; E-mail: g.augusti@libero.it

Received September 18, 2015; Accepted October 26, 2015; Published November 05, 2015

Citation: Augusti D, Gabriele A, Francesca C, Dino R (2015) Does Sandblasting Improve Bond Strength between Nano-ceramic Resin and Two Different Luting Composites? Bioceram Dev Appl 5: 086. doi:10.4172/2090-5025.1000086

Copyright: $\odot 2015$ Augusti D, et al. This is an open-access article distributed under the terms of the Creative Commons Attribution License, which permits unrestricted use, distribution, and reproduction in any medium, provided the original author and source are credited. 


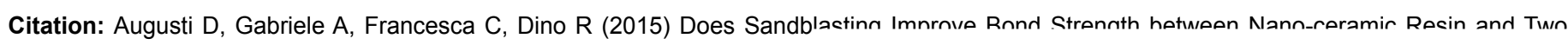
Different Luting Composites? Bioceram Dev Appl 5: 086. doi:10.41

Page 2 of 5

restoration [16]. Nevertheless, technology helped developing adhesive luting materials that are able to ensure a long-lasting and predictable bond between tooth and restoration, improving the retention and reducing the marginal gap, thus preventing the onset of micro-leakage and secondary caries $[17,18]$. Several pre-treatments of the intaglio (i.e: inner side) surface of indirect restorations were developed in order to improve the effectiveness and stability of the bond between resin luting cements and prosthetic materials: for example, the increase of the adhesive area, surface activation, and/or silica-coating plus silanization are main objectives of sandblasting and tribochemical pre-treatments [19]. The above-mentioned procedures applied to the cementation surface would promote penetration of the luting agent and the establishment of micro-mechanical retention [20]. Some Authors stated that sandblasting is able to increase the shear bond strength of luting agents to high-strength ceramics with respect to untreated surfaces, for both traditional composite cements and self-adhesive cements containing functional primers [21-23]. For dental materials containing zirconia, alternative surface treatments have been proposed like the Selective Infiltration Etching (SIE) [24] or a corrosion-controlled process induced by an hot experimental etching solution containing hydrochloric acid (Hot Etching Technique) [25]; usually they can't be easily applied in a chair-side or dental laboratory environment. Sandblasting still remains the most familiar procedure for technicians. While a large amount of information is available for the conditioning of high-strength ceramics, it is still unclear whether sandblasting has a positive influence on the shear bond strength between RNC materials and luting agents. In fact, a number of studies on the bond strength between CAD/CAM materials and resin composites have shown that, besides surface roughening, an additional application of adhesive system might be required $[21,26]$.

\section{Objective}

The aim of this study was to evaluate the effect of sandblasting on the bond strength between RNC blocks and two different luting composites: a self-adhesive dual-cure resin cement (Panavia SA Cement, Kuraray, Tokyo, Japan) and a dual-cure resin cement (RelyX ${ }^{\mathrm{TM}}$ Ultimate, 3M ESPE, Seefeld, Germany).
The null hypothesis was that sandblasting would increase the bond strength values of both luting composites to the RNC material.

\section{Materials and Methods}

\section{Specimens preparation}

$40 \mathrm{CAD} / \mathrm{CAM}$ nano-ceramic resin blocks (Lava ${ }^{\circledR}$ Ultimate, $3 \mathrm{M}$ ESPE, Seefeld, Germany) were selected for this study. All specimen surfaces were milled using Cerec 4.2 compact mill unit burs (Step Bur 12-Ref. 6052265; Sirona Dental GmbH, Austria). The blocks were then ultrasonically cleaned in de-ionized water for 20 minutes and randomly divided into two groups $(\mathrm{n}=20)$ accordingly to the surface treatment that will be applied:

- Group 1 [S_50]: sandblasting performed perpendicularly to the ceramic surface by means of $50 \mu \mathrm{m}$ aluminum- oxide particles $\left(\mathrm{Al}_{2} \mathrm{O}_{3}\right)$ applied for $10 \mathrm{~s}$ at a working distance of $10 \mathrm{~mm}$ and a pressure of $0.28 \mathrm{MPa}$.

- Group $2[\mathrm{C}]$ : no surface treatment.

Each group was further divided into 2 subgroups $(\mathrm{n}=10)$ accordingly to the applied luting technique: [C_LP] and [S_50-LP] were luted using self-etch dual-cure resin cement (Panavia SA); on the other hand, [C_LR] and [S_50-LR] were luted using a dual-cure resin cement (RelyX ${ }^{\mathrm{TM}}$ Ultimate) and a self-etching adhesive system (Scotchbond ${ }^{\mathrm{TM}}$ Universal, 3M ESPE).

\section{Luting procedures}

A single operator prepared the samples analyzed in this study.

Standardized metallic rings with $5 \mathrm{~mm}$ inner diameter and $2 \mathrm{~mm}$ height were filled with a single increment of composite resin (Filtek ${ }^{\mathrm{TM}}$ Supreme XTE, 3M ESPE.). The top of each sample was polymerized for $40 \mathrm{~s}$ at $800 \mathrm{~mW} / \mathrm{cm}^{2}$ with a LED LCU (Bluephase G8, Ivoclar Vivadent, Schaan, Liechtenstein), keeping the light tip orthogonal to the cylinder and in contact with the surface of the composite. Polyethylene adhesive discs (inner diameter $5 \mathrm{~mm}$ ) were employed in order to define the bonding area. Subsequently, the composite resin cylinders were bonded to the nano-ceramic specimens using two different luting cements, employed according to the manufacturer's instructions: half of the

\begin{tabular}{|l|l|l|l|}
\hline Material & Composition & Physical properties & Manufacturer \\
\hline Lava Ultimate & $\begin{array}{l}\text { Nanoparticles of zirconia adherent to } \\
\text { silica: } 80 \%\end{array}$ & Flexural Strength in a dry field: $240 \mathrm{MPa}$ & $3 \mathrm{M}$ ESPE \\
\hline & Resinous material: $20 \%$ & Flexural Strength in a wet field: $230 \mathrm{MPa}$ \\
\hline & Fracture strenght: $200 \mathrm{Mpa} \mathrm{m} \mathrm{m}^{0.5}$ \\
\hline & Resilient: $1.6 \mathrm{MPa}$ \\
\hline & Elastic modulus: $12.77 \mathrm{MPa}$ & N429469 \\
\hline
\end{tabular}

Table 1: Chemical composition and physical properties of Lava Ultimate.

\begin{tabular}{|l|l|c|c|}
\hline Material & \multicolumn{1}{|c|}{ Composition } & Manufacturer \\
\hline Scotchbond ${ }^{\text {TM }}$ Universal (Total-Etch/Self- & Silane & $3 \mathrm{M}$ ESPE \\
\hline Etch, One step) & Initiators & \\
& Water & \\
& Ethanol & \\
& Filler & \\
& VitrebondTM Copolymer & \\
& HEMA & \\
& Dimethacrylate resins & \\
& MDP Phosphate Monomer & \\
\hline
\end{tabular}

Table 2: Composition of Scotchbond Universal. 
Citation: Augusti D, Gabriele A, Francesca C, Dino R (2015) Does Sandblasting Improve Bond Strength between Nano-ceramic Resin and Two Different Luting Composites? Bioceram Dev Appl 5: 086. doi:10.4172/2090-5025.1000086

Page 3 of 5

\begin{tabular}{|c|c|c|c|}
\hline Material & Composition & Manufacturer & Batch \\
\hline RelyX'M Ultimate & $\begin{array}{l}\text { PASTE A } \\
\text { Stabilizers } \\
\text { Initiator components } \\
\text { Radiopaque silanated fillers } \\
\text { Methacrylate monomers } \\
\text { Rheological addictives } \\
\\
\text { PASTE B } \\
\text { Methacrylate monomers } \\
\text { Radiopaque Alkaline (basic) fillers } \\
\text { Silanated fillers } \\
\text { Initiator components } \\
\text { Stabilizers } \\
\text { Pigments } \\
\text { Rheological addictives } \\
\text { Fluorescence dye } \\
\text { Dark cure activator for Scotchbond Universal adhesive }\end{array}$ & 3M ESPE & 540286 \\
\hline Panavia SA Cement & $\begin{array}{l}\text { PASTE A } \\
\text { 10-Methacryloyloxydecyl dihydrogen phosphate (MDP) } \\
\text { Bisphenol A diglycidylmethacrylate (Bis-GMA) } \\
\text { Triethyleneglycol dimethacrylate } \\
\text { Hydrophobic aromatic dimethacrylate } \\
\text { Silanated barium glass filler } \\
\text { Silanated colloidal silica } \\
\text { dl-Camphorquinone } \\
\text { Benzoyl peroxide } \\
\text { Initiators } \\
\text { PASTE B } \\
\text { Bisphenol A diglycidylmethacrylate (Bis-GMA) } \\
\text { Hydrophobic aromatic dimethacrylate } \\
\text { Hydrophobic aliphatic dimethacrylate } \\
\text { Silanated barium glass filler } \\
\text { Silanated colloidal silica } \\
\text { Surface treated sodium fluoride } \\
\text { Accelerators } \\
\text { Pigments }\end{array}$ & Kuraray Medical Ltd & 41117 \\
\hline
\end{tabular}

Table 3: Composition of the luting material employed in the study.

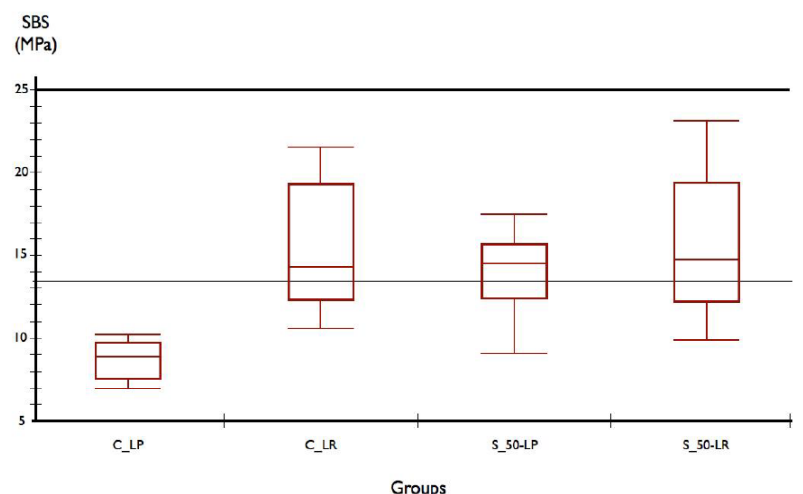

Figure 1: Graphical representation (Boxplot) of obtained SBS values [X axis: Surface treatment; $Y$ axis: Shear bond strength values (MPa)]. Means $(\mathrm{MPa})$ and standard deviations (SD) for the experimental groups were: [CLP]: $8.68 \pm 1.16$; [C-LR]: $15.32 \pm 3.93$; [S_50-LP]: $13.91 \pm 2.58$; [S_50LR]: $15.77 \pm 4.12$. A statistically significant difference $(p<0.001)$ was found between [C-LP] and the other three groups.

samples were luted with Relyx Ultimate, associated with Scotchbond Universal, while half were luted with Panavia SA.

Nano-ceramic-to-composite pressure was obtained with a clamp device, in order to maintain the constant force of $50 \mathrm{~N}$ for the time recommended by manufacturer to achieve an optimal self-curing. Thus, the bonded specimens were store in distilled water at $37^{\circ} \mathrm{C}$ for 1 week. Table 1 shows the chemical composition and physical properties of Lava Ultimate. Tables 2 and 3 include the chemical composition of the adhesive and the cements used in this study, respectively.

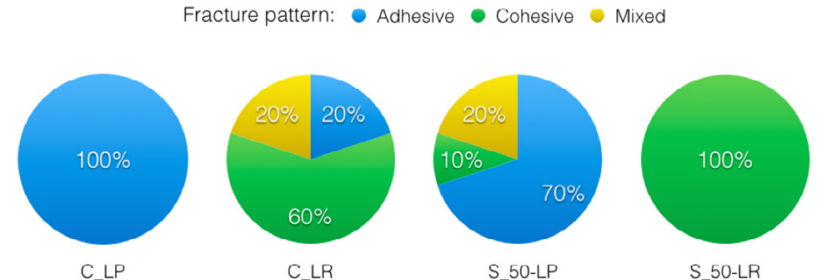

Figure 2: Fracture analysis after SBS test. Specimens luted with $P$ mainly showed adhesive fractures (100\% C_LP; $70 \%$ S_50-LP). Specimens luted with $\mathrm{R}$ showed mainly cohesive fractures (60\% C_LR; $100 \%$ S_50-LR).

\section{SBS test}

A universal testing machine (Instron) was used to determine the shear bond strength at a crosshead speed of $0.5 \mathrm{~mm} / \mathrm{min}$. Before loading, the notched-edge blade was positioned parallel to the adhesive interface. A notched-edge macro shear bond strength test was carried out according to ISO dental standards (ISO 29022:2013). Bond strength values, electronically recorded when the composite - washers debonding occurred, were converted into Mega Pascal (MPa) using the following formula: $\sigma=\mathrm{L} / \mathrm{A}$

Where $\mathrm{L}$ is the load of failure $(\mathrm{N})$ and $\mathrm{A}$ the bonding area $\left(\mathrm{mm}^{2}\right)$.

Measurements were then processed by means of SPSS ${ }^{\circ} 17$, Mac OS X. 


\section{Mode of failure}

A single observer examined fractured interfaces of the specimens with a stereomicroscope (Leica MZ12, Weitzlar, Germany) at 25X magnification, in order to distinguish the failure modes that were defined as follows:

- pure adhesive, when no luting agent residue was found at the ceramic surface

- pure cohesive, when all the bonding area of the ceramic specimen was covered by residuals of luting agent or the fracture was all within the zirconia layer;

- Mixed, when some areas of the bonding surface of the ceramic were covered by the luting agent or a fracture of the outer layer of zirconia occurred.

\section{Statistical analysis}

The means of each group were analysed with SPSS ${ }^{\star} 17$, Mac OS X. considering the shear bond strength as a dependent variable and the types of surface treatment as independent variable. Furthermore, oneway ANOVA test $(\alpha=.01)$ was used to establish differences between the groups.

\section{Results}

Shear bond strength (SBS) values obtained for the experimental groups are graphically represented in Figure 1. Lowest SBS values were obtained for group [C-LP] (minimum-maximum range: 6.92 $10.14 \mathrm{MPa}$ ), while highest adhesive strengths were recorded for group [S_50-LR] (minimum-maximum range: 9.88-23.03 MPa). Statistically significant differences between SBS mean values for groups [C-LP] and [S_50-LP] were observed $(\mathrm{p}<0.001)$. Group $[\mathrm{C}-\mathrm{LP}]$ was significantly different from [C-LR]; conversely, groups [C-LR] and [S_50-LR] presented comparable SBS values (15.33 Vs $15.77 \mathrm{MPa}$, respectively; $\mathrm{p}=0,8)$. Significant differences were not found in groups [S_50-LP] and [S_50-LR] $(\mathrm{p}=0,2)$. Figure 2 shows the results of fractured interfaces analysis observed with a stereomicroscope (Leica MZ12) at 25x magnification. Specimens luted with Panavia SA cement showed $100 \%$ of adhesive fractures for the control group; after sandblasting, cohesive fractures $(20 \%)$ and mixed fractures (10\%) were observed. The fracture pattern of specimens luted with RelyX ${ }^{\mathrm{TM}}$ Ultimate was $60 \%$ of cohesive fractures, $20 \%$ of adhesive fractures and $20 \%$ of mixed fractures in the control group; all samples showed cohesive failure after sandblasting.

\section{Discussion}

The achievement of micromechanical retentions and surface activation are two fundamental principles on which the sandblasting procedure is based on. The surface treatment tested in our study has a long history of proven success in dentistry: in fact, sandblasting has been extensively applied to increase the surface irregularity of a wide range of substrates (like alumina ceramics [27], metals [28] and acrylic materials [20]). In the late 1990's, air abrasion was proposed by Kern in order to increase the resin composite bond strength to zirconia [21]. While the sandblasting process might be affected by several parameters (i.e: size of the particles employed, nature of the particles or jet-pressure), it has been shown effective to promote adhesion between different kind of luting agents and 1) densely-sintered high strength zirconia ceramics [22] or 2) composite/resin-based CAD/CAM materials [20]. In our study, we tried to clarify whether the air abrasion process was also effective to enhance adhesion between two different kinds of resin luting agents and a so called "hybrid material" (RNC), which combines the advantages and properties of both ceramics and composites. The present study showed that air abrasion-performed using $\mathrm{Al}_{2} \mathrm{O}_{3}$, medium-sized $(50 \mu \mathrm{m})$ particles with a standardized $(2.8 \mathrm{bar})$ jet pressure-led to a significant increase of the SBS values (MPa) between the self-adhesive luting agent (Panavia SA, Kuraray Inc.) and RNC surfaces, compared to the control/untreated group ([S_50-LP]: $13.91 \pm$ $2.58 \mathrm{Vs}$ [C-LP]: $8.68 \pm 1.16$ ). Despite some differences in the design of the study, Elsaka et al. have also found a substantial effectiveness of the sandblasting treatment on LAVA Ultimate ${ }^{\mathrm{TM}}$, using another dual-cure self-adhesive resin cement (Bifix SE, VOCO, Germany) [11]. Our results are in agreement with those of Frankenberger et al., who suggested a sandblasting treatment for Lava Ultimate ${ }^{\mathrm{TM}}$ that was not affected by the additional application of a silane, as a separate conditioning step; on the other hand, the same authors reported a detrimental effect of an alternative surface treatment using hydrofluoric acid (i.e: which led to lower SBS values) [10]. As suggested by Chen et al., higher bonding values obtained by a surface treatment like sandblasting might also positively affect the fracture resistance of the material itself; according to the authors, the air-abraded $\mathrm{RNC}$ specimens $\left(50 \mu \mathrm{m}, \mathrm{Al}_{2} \mathrm{O}_{3}\right)$ luted on acrylic discs demonstrated higher fracture loads in comparison to polished-only samples, regardless their thicknesses [12]. On the other hand, the positive and significant effect of air abrasion was not observed in samples luted with the dual-cure resin cement (RelyX Ultimate, 3M ESPE) associated with its proprietary adhesive system (Scotchbond Universal, 3M ESPE). In fact, comparable SBS values (MPa) of 15.3 and $15.7(\mathrm{P}>0.5)$ were found for [C-LP] and [S_50LR] groups, respectively. While sandblasting is a physical/mechanical conditioning process, genuine chemical bond is another way of achieving and promoting adhesion between luting agents and indirect restorative materials $[29,30]$. Some specific molecules and/or their associations - like silane and the functional phosphate monomer MDP (10methacryloyloxydecyl-dihydrogen phosphate) - in the composition of the universal adhesive system (Scotchbond UniversalTM) might explain the satisfactory SBS values obtained within LR groups, regardless the air abrasion process. In particular, the silane molecules (Si-O-Si) (not available on the tested self-adhesive cement) are able to react with hydroxyl radical of silica nanoparticles providing siloxane networks through a chemical bonding, and they also form a bond with metacrylate monomer of the resin luting agent [31]. Moreover, silanes are known to increase surface wettability: this improves the penetration of cement and decreases void formation [23]. Amaral et al. and Gokkaya et al. have reported that the bond strength of resin cements to zirconia is positively influenced by the pre-treatment of the surface with different silanes or by using universal adhesives [32,33]. Finally, the application of adhesives containing silane molecules has been suggested for adhesion between a composite restorative material and CAD/CAM resin blocks for repair purposes [34]. In our specifc case, the presence of inorganic particles (i.e: Nanoparticles of zirconia adherent to silica) on the CAD/CAM RNC surface might help to develop a chemical adhesion through the application of a silane coupling agent. An additional, chemical mechanism of adhesion - beyond sandblasting - is related to a specific functional primer: the MDP molecule is a wellstudied phosphate monomer with bonding capabilities to the zirconia surface (and also to other substrates of interest in dentistry, like metal alloys) [35]. It is possible that MDP monomers may bond chemically to the RNC surface due to a reaction between the exposed hydroxyl groups of zirconia nanoparticles and the hydrogen groups of MDP itself [35]. The application of specific chemical primers or universal adhesives as a single surface pre-treatment might be fascinating from a clinical perspective, in the effort of reducing the number of steps of the luting protocols; however, further studies are necessary to investigate bond 
Citation: Augusti D, Gabriele A, Francesca C, Dino R (2015) Does Sandblasting Improve Bond Strength between Nano-ceramic Resin and Two Different Luting Composites? Bioceram Dev Appl 5: 086. doi:10.4172/2090-5025.1000086

degradation under the challenging intraoral conditions [21]. Finally, the results of the present in-vitro study cannot be generalized to other resin cements or types of CAD/CAM hybrid blocks; the performances of RNC material and retention to tooth structures should be extensively evaluated using in-vivo clinical scenarios.

\section{Conclusion}

Within the limitations of this study, relatively high bond strength values to the Resin-Nano-Ceramic (RNC) surface were found testing the applied materials and protocols. Sandblasting of the ResinNano-Ceramic (RNC) surface is recommended when luting with the tested Panavia SATM self-adhesive cement in order to significantly improve bond strength values. The RelyX Ultimate ${ }^{\mathrm{TM}}$ luting agent - in association with its universal adhesive system including silane and a functional primer-developed satisfactory bond strength values to the RNC surface, regardless a sandblasting pre-treatment. The application of specific chemical primers or universal adhesives as a single surface pre-treatment might be fascinating from a clinical perspective, in the effort of reducing the number of steps of the luting protocols; however, further studies are necessary to investigate bond degradation under the challenging intraoral conditions.

\section{References}

1. Ruse ND, Sadoun MJ (2014) Resin-composite blocks for dental CAD/CAM applications. J Dent Res 93:1232-1234.

2. Santos GC, Santos MJ, Rizkalla AS, Madani DA, El-Mowafy O (2013) Overview of CEREC CAD/CAM chairside system. Gen Dent 61: 36-40.

3. Fasbinder DJ (2010) Materials for chairside CAD/CAM restorations. Compend Contin Educ Dent 31: 702-704.

4. Giordano R (2006) Materials for chairside CAD/CAM-produced restorations. J Am Dent Assoc 137 Suppl: 14S-21S.

5. Alt V, Hannig M, Wostmann B, Balkenhol M (2011) Fracture strength of temporary fixed partial dentures: CAD/CAM versus directly fabricated restorations. Dent Mater 27: 339-347.

6. Stawarczyk B, Ender A, Trottmann A, Ozcan M, Fischer J, et al. (2012) Load-bearing capacity of CAD/CAM milled polymeric three-unit fixed dental prostheses: effect of aging regimens. Clin Oral Investig 16: 1669-1677.

7. Lauvahutanon S, Takahashi H, Oki M, Arksornnukit M, Kanehira M, et al (2015) In vitro evaluation of the wear resistance of composite resin blocks for CAD/CAM. Dent Mater J 34: 495-502.

8. Stawarczyk B, Ozcan M, Trottmann A, Schmutz F, Roos M, et al. (2013) Twobody wear rate of CAD/CAM resin blocks and their enamel antagonists. Prosthet Dent 109: 325-332.

9. Awada A, Nathanson D (2015) Mechanical properties of resin-ceramic CAD/ CAM restorative materials. J Prosthet Dent 114: 587-593

10. Frankenberger R, Hartmann VE, Krech M, Kramer N, Reich S, et al. (2015) Adhesive luting of new CAD/CAM materials. Int J Comput Dent 18: 9-20.

11. Elsaka SE (2014) Bond strength of novel CAD/CAM restorative materials to self-adhesive resin cement: the effect of surface treatments. J Adhes Dent 16: 531-540

12. Chen C, Trindade FZ, de Jager N, Kleverlaan CJ, Feilzer AJ (2014) The fracture resistance of aCAD/CAM Resin Nano Ceramic (RNC) and a CAD ceramic at different thicknesses. Dent Mater 30: 954-962.

13. Harada A, Nakamura K, Kanno T, Inagaki R, Ortengren U, et al. (2015) Fracture resistance of computer-aided design/computer-aided manufacturing-generated composite resin-based molar crowns. Eur J Oral Sci 123: 122-129.

14. Monaco C, Caldari M, Scotti R, Group ACR (2015) Clinical evaluation of toothsupported zirconiabased fixed dental prostheses: a retrospective cohort study from the AIOP clinical research group. Int J Prosthodont 28: 236-238.

15. Ueda K, Beuer F, Stimmelmayr M, Erdelt K, Keul C, et al. (2015) Fit of 4-unit FDPs from $\mathrm{CoCr}$ and zirconia after conventional and digital impressions. Clin Oral Investig.
16. Bowen RL (1980) Adhesive bonding of various materials to hard tooth tissues. XXII. The effects of a cleanser, mordant, and polySAC on adhesion between a composite resin and dentin. J Dent Res 59: 809-814.

17. Breschi L, Mazzoni A, Ruggeri A, Cadenaro M, Di Lenarda R, et al. (2008) Dental adhesion review: aging and stability of the bonded interface. Dent Mater 24: 90-101.

18. Burke FJ, Fleming GJ, Nathanson D, Marquis PM (2002) Are adhesive technologies needed to support ceramics? An assessment of the current evidence. J Adhes Dent 4: 7-22.

19. Re D, Augusti G, Merlati G, Giannì A, Augusti D (2015) Effect of thermal cycling and low-pressure sand-blasting on the bond strength of an self-adhesive cement to Y-TZP zirconia. J Adhes Sci Tech.

20. Soares CJ, Soares PV, Pereira JC, Fonseca RB (2005) Surface treatment protocols in the cementation process of ceramic and laboratory-processed composite restorations: a literature review. J Esthet Restor Dent 17: 224-235.

21. Kern M, Wegner SM (1998) Bonding to zirconia ceramic: adhesion methods and their durability. Dent Mater 14: 64-71.

22. Re D, Augusti D, Augusti G, Giovannetti A (2012) Early bond strength to lowpressure sandblasted zirconia: evaluation of a self-adhesive cement. Eur $J$ Esthet Dent 7: 164-175.

23. Re D, Augusti D, Sailer I, Spreafico D, Cerutti A (2008) The effect of surface treatment on the adhesion of resin cements to Y-TZP. Eur J Esthet Dent 3 : 186-196.

24. Aboushelib MN, Kleverlaan CJ, Feilzer AJ (2007) Selective infiltration-etching technique for a strong and durable bond of resin cements to zirconia-based materials. J Prosthet Dent 98: 379-388.

25. Casucci A, Osorio E, Osorio R, Monticelli F, Toledano M, et al. (2009) Influence of different surface treatments on surface zirconia frameworks. J Dent 37: 891-897.

26. Koller M, Arnetzl GV, Holly L, Arnetzl G (2012) Lava ultimate resin nano ceramic for CAD/CAM: customization case study. Int J Comput Dent 15: 159-164.

27 . Friederich $R$, Kern M (2002) Resin bond strength to densely sintered alumina ceramic. Int J Prosthodont 15: 333-338.

28. Ozcan M, Valandro LF (2012) Bond strength of two resin cements to titanium after different surface Conditioning methods. Gen Dent 60: e6-e12.

29. Lehmann F, Kern M (2009) Durability of resin bonding to zirconia ceramic using different primers. J Adhes Dent 11: 479-483.

30. Stefani A, Brito RB, Kina S, Andrade OS, et al. (2015) Bond Strength of Resin Cements to Zirconia Ceramic Using Adhesive Primers. J Prosthodont.

31. Matinlinna JP, Vallittu PK (2007) Silane based concepts on bonding resin composite to metals. J Contemp Dent Pract 8: 1-8.

32. Amaral M, Belli R, Cesar PF, Valandro LF, Petschelt A, et al. (2014) The potential of novel primers and universal adhesives to bond to zirconia. J Dent 42: $90-98$.

33. Gokkaya FA, Stawarczyk B, Hammerle CH, Sailer I (2013) Influence of silanes on the shear bond strength of resin cements to zirconia. Quintessence Int 44: 591-600.

34. Zaghloul H, Elkassas DW, Haridy MF (2014) Effect of incorporation of silane in the bonding agent on the repair potential of machinable esthetic blocks. Eu J Dent 8: 44-52.

35. Ozcan M, Bernasconi M (2015) Adhesion to zirconia used for denta restorations: a systematic review and meta-analysis. J Adhes Dent 17: 7-26. 